$10 \mathrm{mg} /$ day at presentation. Hydroxychloroquine had been stopped because of ineffectiveness after 15 weeks of use, azathioprine had been stopped due to elevated liver transaminases after 9 weeks of use, intravenous immunoglobulin had been given once at $2 \mathrm{~g} / \mathrm{kg}$ body weight with serological improvement of myositis, mycophenolate mofetil had been stopped due to leg edema after 2 weeks of use, methotrexate was introduced for 3 weeks and stopped because of renal insufficiency. Several series of iloprost infusions were given combined with pentoxifylline orally. Rituximab was applied twice. Sildenafil had been started at $75 \mathrm{mg}$ per day 21 weeks before presentation and was increased to $150 \mathrm{mg}$ per day 17 weeks before presentation.

Based on sporadic reports of phototherapy with improvement of cutaneous affection in connective tissue diseases, we started phototherapy using bath PUVA at a dose of $0.10 \mathrm{~J} / \mathrm{cm}^{2} 3$ times per week, increasing to a maximum single dose of $8 \mathrm{~J} / \mathrm{cm}^{2}$. After a total of 16 weeks of bath PUVA phototherapy with a cumulative dose of $103.7 \mathrm{~J} / \mathrm{cm}^{2}$, a marked improvement in symptoms was noted in the left $3^{\text {rd }}$ and $4^{\text {th }}$ fingers, with only hyperkeratotic plaques remaining, while the lesions on the left $2^{\text {nd }}$ finger and the right hand were stationary. Treatment was continued for a total of 29 weeks with a cumulative dose of $272.3 \mathrm{~J} / \mathrm{cm}^{2}$ bath PUVA, resulting in significant improvement. The necrosis in all fingers disappeared with a remaining isolated crust on the left $4^{\text {th }}$ finger, without new lesions and with improved sensation of both hands (figure 1B).

Follow-up at five months after treatment with bath PUVA revealed slight swelling of both hands without new fingertip lesions.

Patients positive for anti-PM-Scl-70 antibodies suffer from digital ulcers and lung fibrosis more frequently than anti$\mathrm{PM} / \mathrm{Scl}$ antibody-negative patients [2]. For digital ulcers, the EULAR recommendations include prostanoids, in particular, iloprost for therapy and bosentan for prevention [3]. A selective inhibitor of cyclic guanosine monophosphate sildenafil - has shown potential in treating digital ulcers. In our patient, however, vasoactive treatment had been established before phototherapy for seven months with stationary ulcers [4].

While no reports on phototherapy in anti-PM-Scl-70 positive patients exist, other collagen vascular diseases such as SLE have been improved by phototherapy with UV, in particular UVA1 [5]. In those patients, it is believed that the UV light was immunomodulatory and antifibrotic [6]. Based on these similarities, we performed topical phototherapy with bath PUVA in our patient.

Our case showed a dramatic improvement of the local skin condition under concurrent phototherapy in combination with $150 \mathrm{mg}$ sildenafil daily. While we lack any specific immunological data, we believe that a beneficial effect of topical PUVA phototherapy combined with high-dose sildenafil [4] is likely in this patient. It should be noted, however, that spontaneous healing of ulcers can be observed in up to $80 \%$ of patients over 16 weeks [7]. Controlled studies will help to better determine the impact of topical PUVA phototherapy in anti-Pm-Scl-70 antibody-positive SSc-Polymyositis overlap syndrome.

Disclosure. Financial support: none. Conflict of interest: none.
${ }^{1}$ Department of Dermatology,

${ }^{2}$ Department of Rheumatology,

University Hospital Zurich,

Gloriastrasse 31, 8091

Zurich, Switzerland

<mohanna.mosabtariq@usz.ch>
Mosab MOHANNA ${ }^{1}$

Oliver DISTLER ${ }^{2}$

Haiko SPROTT ${ }^{2}$

Thomas KÜNDIG ${ }^{1}$

Lars E. FRENCH ${ }^{1}$

Günther HOFBAUER ${ }^{1}$

1. Distler JH, Kalden JR, Distler $O$. Undifferentiated connective tissue diseases, overlap syndromes, and mixed connective tissue disease. In: $\mathrm{Bij}$ sma JWH, ed. EULAR compendium on rheumatic diseases. 1 st edn. London: BM, 2009: 244-56.

2. Hanke K, Bruckner CS, Dahnrich $C$, et al. Antibodies against PM/Scl-75 and PM/Scl-100 are independent markers for different subsets of systemic sclerosis patients. Arthritis Res Ther 2009; 11: R22.

3. Kowal-Bielecka O, Landewe R, Avouac J, et al. EULAR recommendations for the treatment of systemic sclerosis: a report from the EULAR Scleroderma Trials and Research group (EUSTAR). Ann Rheum Dis 2009; 68: 620-8.

4. Brueckner CS, Becker $M O$, Kroencke $T$, et al. Effect of sildenafil on digital ulcers in systemic sclerosis: analysis from a single centre pilot study. Ann Rheum Dis 2010; 69: 1475-8.

5. McGrath H Jr.. Ultraviolet-A 1 irradiation decreases clinical disease activity and autoantibodies in patients with systemic lupus erythematosus. Clin Exp Rheumatol 1994; 12: 129-35

6. Kroft EB, Berkhof NJ, van de Kerkhof PC, Gerritsen RM, de Jong $E M$. Ultraviolet A phototherapy for sclerotic skin diseases: a systematic review. J Am Acad Dermatol 2008; 59: 1017-30.

7. Korn JH, Mayes $M$, Matucci Cerinic $M$, et al. Digital ulcers in systemic sclerosis: prevention by treatment with bosentan, an oral endothelin receptor antagonist. Arthritis Rheum 2004;50: 3985-93.

doi:10.1684/ejd.2013.2136

\title{
Detection of the deletion of the STS gene and flanking sequences using polymerase chain reaction in a Chinese pedigree: the first case report of $X$-linked ichthyosis asso- ciated with testicular microlithiasis
}

We report a 19-year-old patient (III10) from a fourgeneration Chinese pedigree with desquamation covering almost all the body since about 10 months of age. The development of cutaneous lesions initially occurred on the extremities and, with aging, progressively spread to the trunk, scalp, neck and face. There were no abnormalities of the hands, feet, hair or nails. Scale formation usually appeared to be reduced in warm weather and exacerbated in cold weather. In time, large, thick, adherent, dark-grey scales, preferentially distributed on the trunk and limbs, became more prominent. The abdomen was more severely affected than other parts of the body (figure 1A).

On questioning his family history (figure $1 B$ ), his halfbrother (III8) was found to suffer from the same condition and had classical skin features of ichthyosis. Furthermore, 


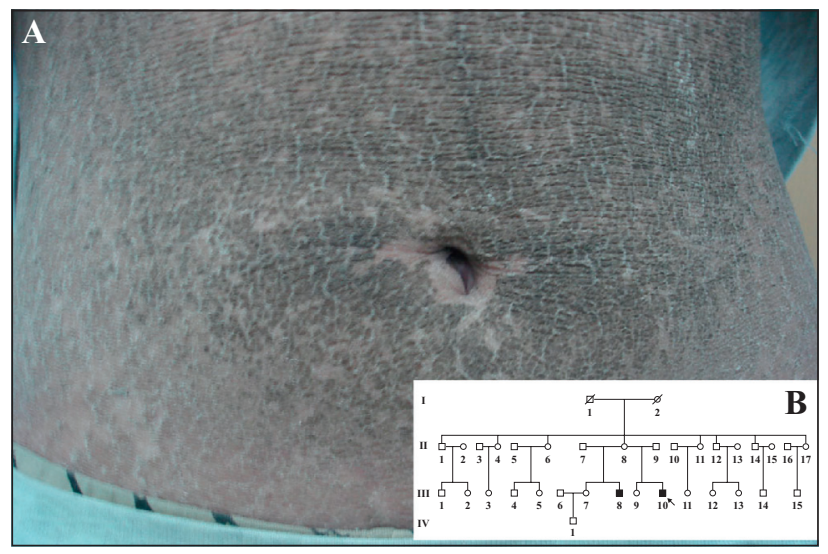

Figure 1. A) Typical clinical lesions of the XLI proband with testicular microlithiasis. Large, thick, tightly adherent, arid, dark-grey scales preferentially distributed symmetrically on the abdomen, the most severely affected around the umbilicus. B) A Chinese four-generation pedigree had two half-brother patients with severe XLI and one obligate mother with mild lesions among all the 35 family members (man/female:18/17).

the mother (II8) of the two half-brothers also presented with mild lesions of ichthyosis, only on the extensor surfaces of the extremities. This mother of four children had neither history of abortion nor complications of delivery. There were no consanguineous marriages among their three parents. No signs of ichthyosis were disclosed in other individuals of pedigree.

Physical examinations of the two half-brothers showed no micro-penises, small testes, cryptorchidism or testicular tumors; ophthalmologic examination showed no corneal opacities or other abnormalities. The growth, development and intellect of the two brothers were normal.

Laboratory investigations of the electromobility rates of beta-lipoprotein electrophoresis revealed no abnormalities. Longitudinal images of the two half-brothers through ultrasound scanning both indicated the typical appearance of testicular microlithiasis, with at least five microliths per sonogram. But the other family individuals showed no abnormalities. Chromosomal analysis, using G-banding, for the two patients and their three parents revealed a 46 , XY karyotype.

\section{PCR and DNA deletion analysis}

The patients and their family were informed about the details of this study and their consent to participation was obtained. Total genomic DNA from peripheral blood leukocytes was extracted. At first, we designed 2 couples of primers flanking the 5'-STS and 3'-STS. The primer for the 5' end is F-ccaggatcacctgaaactcc and R-atgcaacacaagcaggtagc, and the 3 ' end is Ftggtaatcttccatccactgc and R-cattggaaaggctccatt. Then, to detect the extent of DNA deletion, 8 sets of primers for the STS and its flanking regions (telomericDXS89, DXS1139, DXS1130, 5' -STS, 3'-STS, DXS1133, DXS237, DXS1132, DXF22S1 and DXS278-centromeric) were followed by their sequences to amplify according to the report by Schaefer et al. [1,2]. In the two half-brothers, initial PCR analysis revealed no amplification of either end of STS. Moreover, the regional markers from telomericDXS1139 to DXF22S1-centromeric were not amplified.

Hence, these typical clinical presentations, family analyses, laboratory tests and PCR analyses established the diagnosis of X-linked ichthyosis associated with testicular microlithiasis.

$\mathrm{X}$-linked ichthyosis (XLI) is a common genetic disorder, clinically characterized by generalized desquamation, adherent, dark brown scales of the skin, with possible extracutaneous manifestations $[3,4]$. It is caused by a mutation of the steroid sulphatase gene (STS). About $90 \%$ of XLI patients have complete deletion of the entire STS and flanking sequences [5-7]. Depending on the extent of the DNA deletion of Xp22.3, a patient may have XLI either isolated or also with relevant phenotypical anomalies. The contiguous gene deletion syndromes include those for Kallmann syndrome (KAL1), short stature homeobox (SHOX), mental retardation, and ocular albinism (OA1) [4, 8, 9].

Our two patients showed no PCR amplification of the STS and the polymorphism markers, from telomeric-DXS1139 to DXF22S1-centromeric, were approximately $25.7 \mathrm{Mbp}$ in size, according to the NCBI Map Viewer. These breakpoint sites are the most frequent pattern of complete deletion previously described [1, 2, 4-7]. Fortunately, the Kallman gene locates outside that range. Therefore, our pedigree ruled out a Kallmann syndrome and other contiguous gene syndromes.

An increased incidence of testicular maldescent, abnormalities of sperm count or motility and testicular cancer have been reported in patients with XLI [3]. Interestingly, cooccurrence of XLI with testicular microlithiasis for the two patients was definitely diagnosed in our study. No cases of co-existence of these two conditions in the Chinese and English medical literature have been reported until now. Whether the lithogenesis mechanism of biochemistry in the testes is associated with abnormality of the steroid and lipid metabolism needs to be clarified.

Disclosure. Financial support: This study was supported by the National Natural Science Foundation of China (No. 30500537, 30972800 and LY12H11011). Conflict of interest: none.

$\begin{array}{lr}\text { First Affiliated Hospital, } & \text { XueQi ZHANG } \\ \text { Wenzhou Medical College, } & \text { JingJing LIU } \\ \text { No 1 Fuxue lane, } & \text { XiaoHong SHAO } \\ 325000 \text { Wenzhou, } & \text { JianFeng CAI } \\ \text { Zhejiang, China } & \text { ShenMeng GAO } \\ \text { <tigar@aliyun.com> } & \text { XiaoHua LIN } \\ & \text { YueYue YUAN } \\ & \text { ZhiMing LI } \\ \text { BingXu LI } \\ \text { YunSheng XU }\end{array}$

1. Saeki H, Kuwata S, Nakagawa H, Shimada S, Tamaki K, Ishibashi $Y$. Deletion pattern of the steroid sulphatase gene in Japanese patients with X-linked ichthyosis. Br J Dermatol 1998; 139: 96-8. 
2. Jimenez Vaca AL, Valdes-Flores Mdel R, Rivera-Vega MR, GonzálezHuerta LM, Kofman-Alfaro SH, Cuevas-Covarrubias SA. Deletion pattern of the STS gene in X-linked ichthyosis in a Mexican population. Mol Med 2001; 7: 845-9.

3. Traupe $H$, Happle R. Clinical spectrum of steroid sulfatase deficiency: X-linked recessive ichthyosis, birth complications and cryptorchidism. Eur J Pediatr 1983; 140: 19-21.

4. Ballabio $A$, Bardoni $B$, Carrozzo $R$, et al. Contiguous gene syndromes due to deletions in the distal short arm of the human $X$ chromosome. Proc Natl Acad Sci 1989; 86: 10001-5.

5. Bonifas JM, Morley BJ, Oakey RE, Kan YW, Epstein EH Jr.. Cloning of a cDNA for steroid sulfatase: frequent occurrence of gene deletions in patients with recessive $X$ chromosome-linked ichthyosis. Proc Natl Acad Sci U S A 1987; 84: 9248-51.

6. Shapiro L, Yen P, Pomerantz D, Martin E, Rolewic L, Mohandas T. Molecular studies of deletions at the human steroid sulfatase locus. Proc Natl Acad Sci U S A 1989; 86: 8477-81.

7. Cuevas-Covarrubias SA, Kofman-Alfaro SH, Maya-Núñez G, DíazZagoya JC, Orozco Orozco E. X-linked ichthyosis in Mexico: high frequency of deletions in the steroid sulfatase encoding gene. Am J Med Genet 1997; 72: 415-6.

8. Cuevas-Covarrubias SA, González-Huerta LM. Analysis of the VCX3A, VCX2 and VCX3B genes shows that VCX3A gene deletion is not sufficient to result in mental retardation in X-linked ichthyosis. $\mathrm{Br}$ J Dermatol 2008; 158: 483-6.

9. Hernández-Martín A, González-Sarmiento $R$, De Unamuno P. X-linked ichthyosis: an update. $\mathrm{Br} J$ Dermatol 1999;141: 617-27.

doi:10.1684/ejd.2013.2121

\section{Impact of vitiligo on the health-related quality of life of 104 adult patients, using Dermatology Life Quality Index and stress score: First Egyptian report}

\footnotetext{
Despite the large number of vitiligo patients in our rapidlyexpanding population, no studies are available addressing the health-related quality of life (QOL) of vitiligo patients in our community. The current study verified the healthrelated QOL in vitiligo patients in our country, with its peculiar settings, using both the Dermatology life quality index (DLQI) and the stress score (SS).

The current prospective study included all adult patients with a clinical diagnosis of vitiligo attending the dermatology outpatient clinic, Cairo University, (January 2011 -February 2012) $(\mathrm{n}=104)$, serving mainly Giza governate, population 3,326,444. Patients were off treatment to avoid the impact of different therapeutic lines on the healthrelated QOL. Any patient with evidence of psychological, systemic or another dermatological disorders was excluded. 108 healthy, age, sex and socioeconomically matched controls were also included.

All participants answered a preset questionnaire to document their demographic data, disease duration and activity, according to the Vitiligo Disease Activity (VIDA) Score [1]. Patients were examined to document their skin phototypes, disease visibility and Vitiligo Area Scoring Index (VASI) [2].
}

Validated Arabic versions of DLQI [3] (for patients) and SS [4] (for patients and controls) were used. The last question in DLQI was omitted according to the designed exclusion criteria. DLQI $>10$ indicated a very great health-related QOL impairment, DLQI (6-10) indicated a moderate effect, DLQI (2-5) indicated little effect and DLQI 0 or 1 indicated no effect on health-related QOL [5].

The demographic data and clinical parameters of patients are summarized in table 1. The total scores of DLQI among vitiligo patients ranged between 1 and $24(9.52 \pm 5.88)$. The highest DLQI was achieved in DLQI 8, 9 (0-6, 3.42 \pm 1.74$)$, then DLQI 1, $2(0-5 ; 2.31 \pm 1.089)$, DLQI 3, 4 (0-6; $1.71 \pm 2.03)$, DLQI $5,6(0-6,1.38 \pm 1.942)$ and finally DLQI $7(0-3,0.66 \pm 1.07) .44$ patients $(42.3 \%)$ had DLQI $>10,28(26.9 \%)$ had DLQI 6-10, $26(25 \%)$ had DLQI $2-5$ and $6(5.8 \%)$ had DLQI $0-1$. The SS among patients $(12-35(25.37 \pm 5.86))$ was significantly higher than controls $(7-34 ; 16.81 \pm 8.29)(\mathrm{P}=0.045)$. Multivariate logistic regression analysis revealed that skin phototype $(-0.23$, $0.007)$, education $(0.247,0.02)$, acrofacial presentation $(0.235,0.007)$ and SS $(0.402,0.00)$ are the most important variables that significantly influence the DLQI, whereas age $(-0.318,0.001)$, VIDA $(0.319,0.000)$ and DLQI $(0.376$, 0.000 ) are the most important variables that influence the SS.

The negative impact that vitiligo exerts on the health-related QOL of adult Egyptian patients was clearly shown in the current study, as described by the results of the DLQI as well as the SS, and was significantly higher in vitiligo patients. The mean total DLQI (9.52) documented in the current study is among the highest reported scores [6-9]. This could be attributed to the relatively darker skin phototypes, leading to a greater contrast between pigmented and depigmented skin and to the different social, cultural, economic and religious frameworks of our community, which might be translated into a worse health-related QOL.

Despite the documented high DLQI, we believe that it is actually an underestimation, not only because of the omission of DLQI 10, making the maximum achievable score 27 instead of 30, but also because in several situations the answer to a given question was "not relevant" and not "not at all" and in both cases a score of zero was given. In our opinion this raises a problem that, although the DLQI used is a well-known validated instrument dealing with dermatological diseases and their impact on the health-related QOL, each community still has its distinctive social, economic, religious and cultural structures which might necessitate the use of combined different scoring systems to achieve a more accurate assessment of the health-related QOL.

This is the first study to assess the health-related QOL in adult Egyptian vitiligo patients using both DLQI and SS. It revealed a significant deterioration of their healthrelated QOL, with younger, female, educated patients with darker skin phototypes, visible lesions and a positive family history being at higher risk. Even in countries with limited resources like ours, it is important to recognize and address the psychological issues as it would ultimately lead to improved health-related QOL and thereby treatment outcomes, due to the well-known role played by psychological stress in the initiation and progression of vitiligo.

Disclosure. Financial support: none. Conflict of interest: none. 